\title{
High-resolution Spectroscopy of the Nickel-like Molybdenum X-ray Laser Toward the Generation of Circularly Polarized X-ray Laser
}

\author{
Noboru Hasegawa*, Akira Sasaki, Hiroshi Yamatani, Maki Kishimoto, Momoko Tanaka, \\ Yoshihiro Ochi, Masaharu Nishikino, Yuichi Kunieda, and Tetsuya Kawachi \\ Quantum Beam Science Directorate, Japan Atomic Energy Agency (JAEA) \\ 8-1 Umemidai, Kizugawa, Kyoto 619-0215, Japan \\ Hitoki Yoneda \\ Institute for Laser Science, University of Electro-Communications \\ 1-5-1, Chofugaoka, Chofushi, Tokyo 182, Japan \\ Atsushi Iwamae \\ Department of Engineering, Faculty of Engineering, Kyoto University, Kyoto 606-01, Japan
}

(Received December 16, 2008 : revised February 26, 2009 : accepted February 26, 2009)

\begin{abstract}
We attempted the first measurement of the spectral width of the nickel-like molybdenum x-ray laser $(\lambda=18.895 \mathrm{~nm})$ by use of a high-resolution spectrometer in order to determine the strength of the magnetic field required for the generation of a circularly polarized x-ray laser. The spectral width was measured to be $\Delta \lambda=18 \mathrm{~m} \AA$ under the substantial lasing condition. The magnetic field required for the generation of a circularly polarized x-ray laser was $40 \mathrm{~T}$.

The splitting of the x-ray laser line was clearly obtained under $15 \mathrm{~T}$ external magnetic field. The strength of the magnetic field estimated from the splitting of the $\mathrm{x}$-ray laser line was large compared with the external magnetic field. It implies that there might be an alternative mechanism for enhancement of the magnetic field in the gain medium plasma.
\end{abstract}

Keywords : X-ray laser, High-resolution spectroscopy, Circular polarization

OCIS codes : (140.7240) UV, EUV, and X-ray lasers; (300.6320) Spectroscopy, high-resolution

\section{INTRODUCTION}

Polarized light sources are powerful tools for the analysis of the structure of many materials in the wavelengths of the visible and hard x-ray region. In particular, the circular dichroism measurement is very useful to analyze the 3-D structure of the materials, including the classification of the optical isomer (chirality) [1]. In the wavelength of the visible and hard x-ray regions, the phase control device [2] used for the generation of circularly polarized light is the transmission optics. It is difficult to control the polarization in the wavelength

*Corresponding author: hasegawa.noboru@jaea.go.jp of the soft x-ray. Recently, the circularly polarized soft $\mathrm{x}$-ray has been generated by a combination of synchrotron and angulators [3]. In order to generate the circularly polarized soft x-ray by a laboratory scale device, we propose a new approach for the generation of circularly polarized XRL by use of a magnetic field. In this method, the spectral width of XRL is quite an important parameter because the strength of the magnetic field required for the generation of circularly polarized XRL is determined by the spectral width of XRL. Only a few measurements of the spectral width of the XRL have been reported $[4,5]$, because the spectral width of the XRL is so narrow that the required spectral resolution is quite high, e.g. $\Delta \lambda / \lambda \sim 10^{-4}$. Therefore we measured the 
spectral width of XRL using a high-resolution spectrometer, and demonstrated the preliminary experiment for the circularly polarized XRL.

\section{SCHEME FOR THE GENERATION OF CIRCULARLY POLARIZED X-RAY LASER}

We considered the transition of the XRL to generate the circularly polarized XRL. Figure 1 shows the Kastler diagram of the XRL using the nickel-like ion. The XRL is generated between the $4 d(J=0)$ and the partial $4 p(J=1)$ levels. There are three magnetic sublevels with $m_{J}=-1,0,1$ in the lower level, and the XRL has three degenerate lines. The polarization of these lines are left-handed circular $\left(\sigma^{\prime}\right)$, linear $(\pi)$ and right-handed circular polarization $(\sigma)$. The direction of the radiation of the circular components and linear component are parallel and perpendicular to the quantization axis, respectively. If we take the quantization axis to the direction of the external magnetic field, the circularly polarized XRL can be extracted.

The schematic figure of the method of the extraction of the circularly polarized XRL is shown in Fig. 2. If the strength of the magnetic field is large enough to separate each polarization component by the Zeeman effect, each of the circular polarization components can be extracted separately after resolving with a high-resolution spectrometer. The advantage of this method is that the right- and left-handed circularly polarized XRL are obtained at the same time. The Zeeman effect of the degenerate lines of highly charged ions such as XRL line can be treated by the linear Zeeman effect, and the value of the split is described as $2 \Delta \varepsilon=\{J(J$ $+1)\}^{1 / 2} \mu_{B} B\left(=1.6 \times 10^{-4} B \mathrm{eV}\right)$, where $\mu_{B}$ is the Bohr magnetron $\left(=5.8 \times 10^{-5} \mathrm{eV} / \mathrm{T}\right)$, and $B$ is the strength of the magnetic field. To extract each circularly polarized

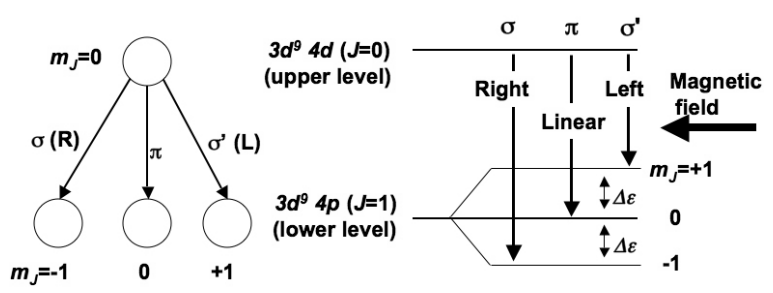

FIG. 1. Kastler diagram of XRL using the nickel-like ions.

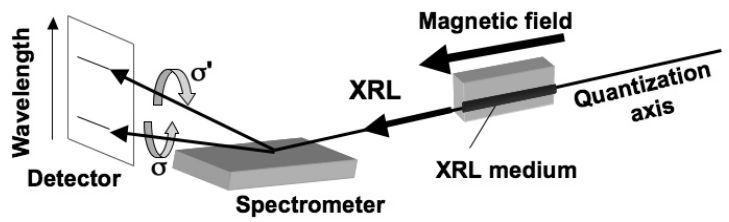

FIG. 2. The schematic figure for the extraction of the circularly polarized XRL.
XRL component, the Zeeman split value has to be larger than the spectral width of XRL without the external magnetic field. Therefore the spectral width of XRL is important parameter to determine the strength of the magnetic field required for the circularly polarized XRL.

In this study, we choose the nickel-like molybdenum XRL $\left(3 d^{9} 4 p{ }^{1} P_{1}-3 d^{9} 4 d^{1} \mathrm{~S}_{0}\right)$ at a wavelength of 18.895 $\mathrm{nm}$ as an example. The reasons for using this laser are as follows: strong amplification has been obtained with the grazing incident pumping scheme (GRIP) [6] in previous work [7], and the wavelength can be covered by a high-resolution spectrometer.

\section{MEASUREMENT OF SPECTRAL WIDTH OF NICKEL-LIKE MOLYBDENUM X-RAY LASER}

The experimental setup is shown in Fig. 3. The nickellike molybdenum XRL was generated from the linearly formed plasma (Ni-like Mo ions) and measured by the highresolution spectrometer, HIREFS made by Hettrick Scientific [8].

We used the GRIP scheme for the focusing system of the Nd:glass laser at a wavelength of $1053 \mathrm{~nm}$ [9]. The GRIP scheme can control the electron density $\left(n_{e}\right)$ of the laser absorption region by changing the grazing angle $(\theta)$ to the target surface. The electron density of the laser absorption region is described to be $n_{e}=n_{c r} \sin ^{2} \theta . n_{c r}$ is the critical electron density, and it is $10^{21} \mathrm{~cm}^{-3}$ for the laser with the wavelength of $1053 \mathrm{~nm}$. The grazing incidence angle was set to 14 degrees, and the electron density of the laser absorption region was $6 \times 10^{19} \mathrm{~cm}^{-3}$. The focal width and length were measured to be $70 \mu \mathrm{m}$ and $5 \mathrm{~mm}$, respectively. Nd:glass laser light consisted of two pulses, the pre-pulse and the main pulse. The total energy on the target was $10 \mathrm{~J}$, and the energy ratio of these pulses was $1: 4$. The time separation between two pulses was $1.1 \mathrm{~ns}$. The durations of the pre-pulse and the main pulse were $7 \mathrm{ps}$ each. The pre-pulse was generated by a simple method; it was generated from the regenerative amplifier of the Nd:glass laser by changing a timing of the pockels cell pulse slicer. Therefore the optical axis and the duration of the pre-pulse were same as the main pulse. The present laser system and GRIP scheme were suitable

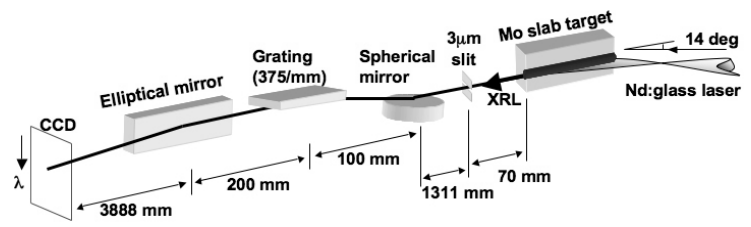

FIG. 3. Experimental setup for the measurement of the spectral width of Ni-like Mo XRL. 
for the generation of the circularly polarized XRL which should set the XRL medium into the small diameter $(\sim 4 \mathrm{~mm})$ magnetic coil (see in section IV).

HIREFS has a spherical mirror and an elliptical mirror to transfer the image on the slit to the detector. The slit was set at the position of $70 \mathrm{~mm}$ from the edge of the plasma, and the width of the slit was $3 \mu \mathrm{m}$. The magnification of the horizontal plane (= the direction of the plasma expansion) and the vertical plane $(=$ the direction of the wavelength dispersion) were 2.4 and 3.2, respectively. The back-illuminated CCD (Princeton, PI-SX:1K, pixel size $13 \mu \mathrm{m})$ was used for the detector. The spatial resolution of HIREFS on the CCD surface in the direction of the wavelength dispersion was $9.4 \mu \mathrm{m}$, and it was smaller than the CCD pixel size. Therefore the resolution in the wavelength was determined by the CCD pixel size. Carbon Balmer $\alpha$ line $(182.20 \AA)$ and Ni-like Mo XRL line $(188.95 \AA)$ [10] were measured to decide the inverse linear dispersion of HIREFS. The inverse linear dispersion was $785 \mathrm{~m} \AA / \mathrm{mm}$ on the CCD surface. The theoretical resolution of HIREFS was estimated to be $10.2 \mathrm{~m} \AA\left(\mathrm{d} \lambda / \lambda=5.4 \times 10^{-5}\right)$.

Figure 4 shows the spectral profiles of the Ni-like Mo XRL line at gain length product $(g l)$ of 3.1 (a), 4.2 (b), and 6.9 (c). Each dot and the solid curves show the data points and the fitting curves, respectively. The details of the fitting curves are described later. The spectral width was measured to be $36 \mathrm{~m} \AA$ (a), $19 \mathrm{~m} \AA$ (b), and $18 \mathrm{~m} \AA$ (c). The spectral width was increasing with smaller value of $g l$ in the range of $g l$ less than 4 . Consequently, the strength of the magnetic field required for the splitting of circular polarization components was estimated to be $40 \mathrm{~T}$. To obtain a $40 \mathrm{~T}$ magnetic field is not difficult using a pulse-power magnet system [11].

The spectral profile of the XRL is determined by the several components, i.e., Stark broadening, Doppler broadening, gain narrowing effect, and re-broadening effect during the amplification. In the present experiment, we considered the Stark broadening, Doppler broadening, and gain narrowing effect because the amplification of XRL was not saturated. The intrinsic spectral profile of the XRL is determined by convolution of the homogeneous and inhomogeneous broadening, e.g. Voigt profile [4]. Homogeneous broadening $\left(=\Delta \omega_{L}\right.$, (full width at half maximum: FWHM)) is due to Stark broadening and it has the Lorentzian profile. Inhomogeneous broade$\operatorname{ning}\left(=\Delta \omega_{D}(\mathrm{FWHM})\right)$ is due to Doppler broadening and it has the Gaussian profile. The intrinsic spectral profile, $f(\omega)$, is described as

$$
f(\omega)=\frac{2 \ln 2 \Delta \omega_{L}}{\pi^{3 / 2} \Delta \omega_{D}} \int_{-\infty}^{+\infty} \frac{\exp \left(-4 \ln 2\left(\omega_{0}-\omega_{\xi}\right)^{2} / \Delta \omega_{D}^{2}\right)}{\left(\omega_{\xi}-\omega\right)^{2}+\left(\Delta \omega_{L} / 2\right)^{2}} d \omega_{\xi} .
$$

$\omega$ and $\omega_{0}$ are angular frequency and angular frequency of the wavelength center, respectively. The Doppler broadening is written by the ion temperature $\left(T_{i}\right)$, ion mass $\left(m_{i}\right)$ and light velocity $(c) ; \Delta \omega_{D} / \omega_{0}=\left(8 \ln 2 k T_{i} /\right.$ $\left.\left(m_{i} c^{2}\right)\right)^{0.5}$ The ion temperature in the present experiment were calculated by the 1-D hydrodynamic code HYADES [12]; $T_{i}=60 \mathrm{eV}$. It implied the Doppler broadening $\left(\Delta \lambda_{D}\right)$ was $12 \mathrm{~m} \AA$. We supposed the spectral profile at $g l=3.1$ to be the intrinsic spectrum of the lasing line without the gain narrowing effect. The spectrum of Fig. 4 (a) was fitted by $f(\omega)$ with $\Delta \lambda_{L}$ of $30 \mathrm{~m} \AA$ and $\Delta \lambda_{D}$ of $12 \mathrm{~m} \AA$. Total spectral width was $36 \mathrm{~m} \AA$.

In Fig. 4 (b) and (c), amplified XRL spectra can be described as the intrinsic spectrum and the gain length product. The amplified XRL spectral profile, $I(\omega, l)$, is described as

$$
I(l, \omega)=f(\omega) \exp \left(g^{\prime} l\right) .
$$

$l$ and $g^{\prime} l$ are the length of the gain medium and the fitting parameter of the amplified XRL spectral profile, respectively. If the gain re-broadening effect can be ignored, $f(\omega)$ is same as the gain profile; $g(\omega)$, and $g^{\prime} l$ can be described as $\{f(\omega) l-3.1\}$ because $f(\omega)$ was
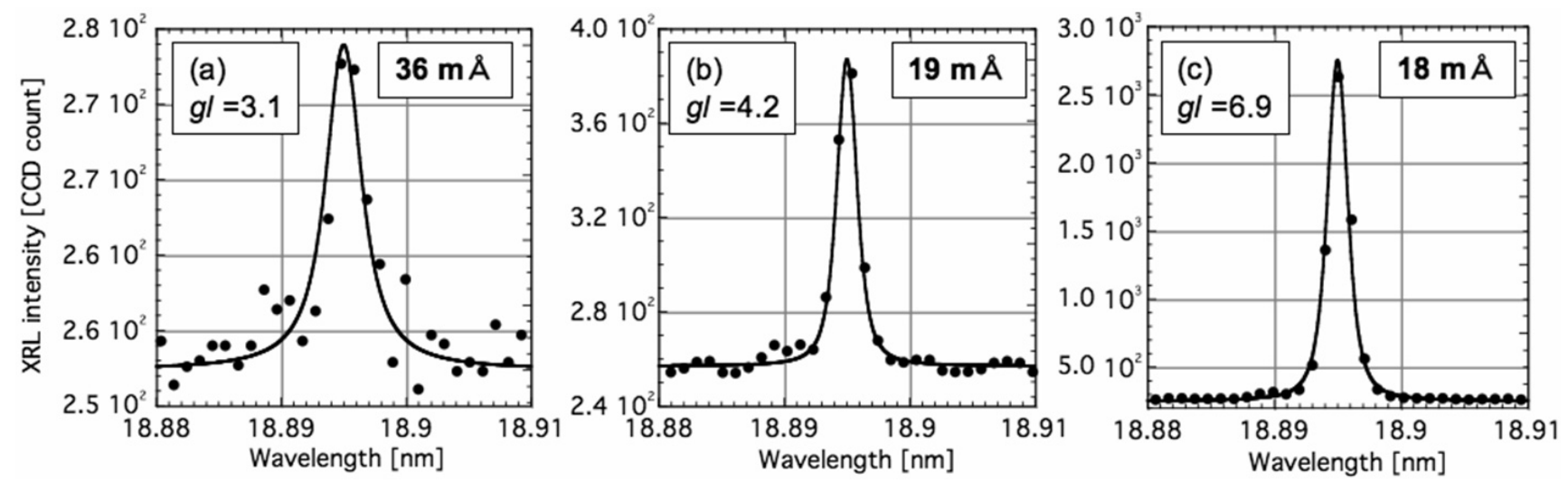

FIG. 4. Ni-like Mo XRL spectral profiles under the condition $g l=3.1$ (a), 4.2 (b), and 6.9 (c). 
obtained at $g l=3.1$ in the result of fitting in Fig. 4 (a). In Fig. 4 (b) and (c), the fitting parameter $\left(g^{\prime} l\right)$ were 2.0 and 2.3 , respectively. The calculated $g l$ from the fitting parameters did not agree with the experimental result of $g l$. These differences might be the cause of a gain re-broadening effect or an instrumental broadening of the HIREFS.

\section{PRELIMINARY RESULT OF GENERATION OF THE CIRCULARLY POLARIZED X-RAY LASER}

We demonstrated the extraction of the circularly polarized Ni-like Mo XRL. The XRL target was the thin rod (the cross section was $1 \mathrm{~mm} \times 1 \mathrm{~mm}$, and the length was $30 \mathrm{~mm}$ ) of molybdenum to set at the center of the electro-magnetic coil. The length and inner diameter of the magnetic coil were $5 \mathrm{~mm}$ and $4 \mathrm{~mm}$, respectively. The peak strength of the external magnetic field was measured to be $15 \mathrm{~T}$ by using the Faraday rotation of the linearly polarized laser. The rotation angle of the polarization of the linearly polarized $\mathrm{Nd}$ : YAG laser at the wavelength of $532 \mathrm{~nm}$ was measured to be 20.5 degrees by passing the magnetic coil which the fused silica inserted in.

The spectral profile of the XRL was measured by HIREFS. Figure 5 shows the obtained spectra of the Ni-like Mo XRL without (a) and with (b) the external magnetic field. The difference between these spectra was clearly seen, and apparent split of the spectrum was obtained under the presence of the magnetic field. As the refraction angle of the XRL increases, the separation becomes larger. If the separation of the spectrum $(2 \Delta \varepsilon)$ was decided only due to the Zeeman effect, i.e., $2 \Delta \varepsilon=1.6 \times 10^{-4} \mathrm{~B} \mathrm{eV}$, the strength of the magnetic field near the target surface was higher than that far from the target surface, and the quantities

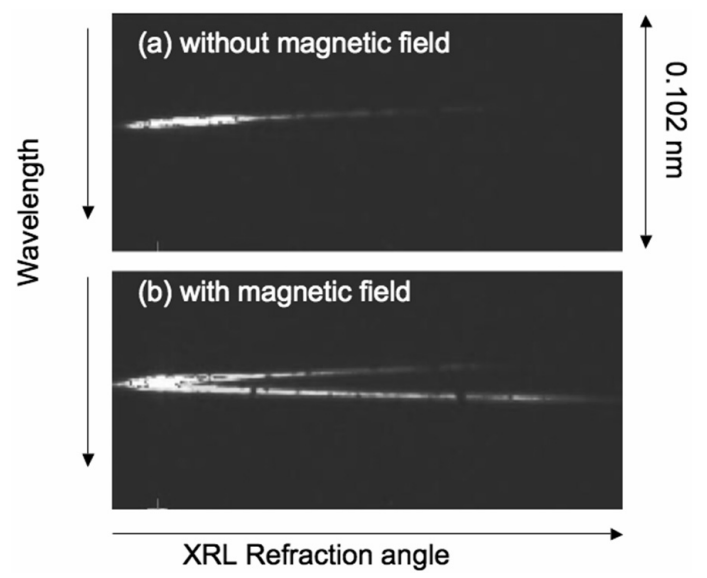

FIG. 5. Ni-like Mo XRL spectra without (a) and with (b) the external magnetic field. were high compared with the external magnetic field. If the strength of the magnetic field in the XRL medium was equal to the external magnetic field, the quantity of the separation of the XRL spectrum was less than half of the XRL spectral width. It implies that there might be an alternative mechanism for enhancement of the magnetic field in plasma, such as the spatial compression of the magnetic flux due to the shock wave that [13] occurred when the main pulse of the pumping laser irradiated the XRL medium. The detail of the mechanism is under consideration now.

\section{SUMMARY}

We have measured the precise spectral width of the Ni-like Mo XRL to determine the strength of the magnetic field for the generation of circularly polarized XRL. The amplified spectral width of the XRL was measured to be $18 \mathrm{~m} \AA$ at the wavelength of $18.895 \mathrm{~nm}$. From this result, the strength of the magnetic field for the generation of circularly polarized x-ray laser was obtained to be $40 \mathrm{~T}$. In addition, a preliminary experiment was conducted under the external magnetic field $(=15 \mathrm{~T})$, and the separation of the XRL line was obtained. The estimated strength of the magnetic field in the XRL medium from the split value of the spectrum of the XRL was much higher than the external magnetic field. This result implied there was an alternative mechanism for enhancement of the magnetic field in plasma, such as the spatial compression of the magnetic flux due to the shock wave.

\section{ACKNOWLEDGMENT}

This work was supported by a Grand-in-Aid for Young Scientists (B) (No. 19740350) from the Japan Society for the Promotion of Science.

We would like to thank Professor Nishimura in Osaka University for the use of HIREFS..

\section{REFERENCES}

1. G. D. Fasman Eds., Circular Dichroism and the Conformational Analysis of Biomolecules (Plenum Press, New York, 1996).

2. K. Hirano, K. Okitsu, A. Momose, and Y. Amemiya, "Recent research related to the polarization and/or phase of SR," Advances in X-Ray Chemical Analysis Japan 33, 25-43 (2001).

3. M. Tanaka, K. Nakagawa, A. Agui, K. Fujii, and A. Yokoya, "First observation of natural circular dichroism for biomolecules in soft x-ray region studied with a polarizing undulator," Phys. Scr. T115, 873-876 (2005). 4. J. A. Koch, B. J. MacGowan, L. B. D. Silva, D. L. 
Matthews, J. H. Underwood, P. J. Batson, R. W. Lee, R. A. London, and S. Mrowka, "Experimental and theoretical investigation of neonlike selenium x-ray laser spectral linewidths and their variation with amplification,” Phy. Rev. A 50, 1877-1898 (1994).

5. N. Hasegawa, T. Kawachi, T. Utsumi, A. Sasaki, M. Tanaka, M. Kado, K. Sukegawa, P. Lu, M. Kishimoto, R. Tai, K. Nagashima, M. Koike, H. Daido, and Y. Kato, "High-precision measurement of the wavelength of a Ni-like silver x-ray laser,” Jpn. J. Appl. Phys. 43, 2519-2522 (2004).

6. R. Keenan, J. Dunn, V. N. Shlyaptsev, R. F. Smith, P. K. Patel, and D. F. Price, "Efficient pumping schemes for high average brightness collisional x-ray lasers," Proc. SPIE 5197, 213-220 (2003).

7. B. M. Luther, Y. Wang, M. A. Larotonda, D. Alessi, M. Berrill, M. C. Marconi, J. J. Rocca, and V. N. Shlyaptsev, "Saturated high-repetition-rate 18.9-nm tabletop laser in nickellike molybdenum,” Opt. Lett. 30, 165-167 (2005).
8. M. C. Hettrick, J. H. Underwood, P. J. Batson, and M. J. Eckart, "Resolving power of 35,000 ( $5 \mathrm{~m} \AA$ ) in the extreme ultraviolet employing a grazing incidence spectrometer,” Appl. Opt. 27, 200-202 (1988).

9. T. Kawachi, M. Kado, M. Tanaka, N. Hasegawa, K. Nagashima, K. Sukegawa, P. Lu, K. Takahashi, S. Namba, M. Koike, A. Nagashima, and Y. Kato, "Development of a pumping laser system for x-ray laser research," Appl. Opt. 42, 2198-2205 (2003).

10. Y. Li, J. Nilsen, J. Dunn, and A. L. Osterheld, "Wavelengths of the Ni-like $4 \mathrm{~d}{ }^{1} \mathrm{~S}_{0}-4 \mathrm{p}{ }^{1} \mathrm{P}_{1} \mathrm{x}$-ray laser line," Phys. Rev. A 58, 2668-2671 (1998).

11. K. Kindo, "100T magnet developed in Osaka," Physica. B 294-295, 585-590 (2001).

12. G. J. Pert, "The hybrid model and its application for studying free expansion,” J. Fluid Mech. 131, 401-426 (1983).

13. F. G. Tomasel, V. N. Shlyaptsev, and J. J. Rocca, "Enhanced beam characteristics of a discharge-pumped soft-x-ray amplifier by an axial magnetic field," Phys. Rev. A 54, 2474-2478 (1996). 